\title{
DORA : The Travel Assistant
}

\author{
Mrs. Aparna kadam, Mrs. Vandana Singh, Mrs. Mayuri mali \\ Guided by : prof. yogita chavan \\ New horizon Institute of Technology and management
}

\begin{abstract}
In this paper we introduce Voice text Command device (VTCD) a universe voice control solution for nonvisual access to the Android operating system. The reader, which transform digital information to text and then to voice by TTS ( Text -To-Speech), is widely used to help visually impaired persons to operates devices. The systems, integrates open source and also enhances many functions of them. It include the message reader, text file reader, OCR reader, voice dialer. In addition, it provide the navigation Reader for walking and riding, which is based on Google maps and supports more detailed voice guidance for the distance to a destination and direction . chat bots are an intelligent system being developed using artificial intelligent ( AI) and natural language processing ( NLP ) algorithms.
\end{abstract}

\section{INTRODUCTION}

A travel assistance Voice Text chatbot interacts on a format similar to instant messaging. While a bot is a computer's ability to understand human text \& speech easily. It allows a interaction between a human $\&$ machine for communication purpose, which happens via messages or voice command. In this application user is able to access the services of smart phone with their voice \& text command.

User can easily send a message \& get the response immediately from the chatbot $\&$ their contact list as well as to the mobile number by voice $\&$ text command. Chapter 1 In this Project physically disable person \& person who are uneducated can also use smartphone or how to access the smart phone can easily access phone with their voice or speech command. This application includes the functions of voice \& text services.

DORA The travel assistant chatbot is nothing but a software that would perform automatic tasks. A bot is a computer's ability to understand human speech or text. A chatbot is a program that is ued to simulates human conversations. DORA would take the input and process it to get the desired output using natural language processing.

\section{REVIEW OF LITERATURE}

In existing system, chatbot takes input in the form of text command through chatbot application, analyses that input using Natural LanguageProcessing techniques to find out what the user is trying to say or ask and responds accordingly. The travel chatbot is built to take allnecessary inputs from the user to predict the relevant and accurate answers to the users.

The chatbot will help the user for tourist purpose .The bot tells about the nearby places like restaurants, hotels. In the existing system the user needs to book tickets by himself. The user hasto do through a login form, enter details, or book tours and travels services. This long and manual process becomes very difficult and time consuming. Lots of planning is required for traveling.

\section{REQUIREMENT ANALYSIS}

1. Functional Requirements

2. Non - Functional Requirements

1. Functional Requirements:

- Understand your users.

- Allow unregistered users to register on the application and

- $\quad$ save their details to the database.

- The Chat bot will assist users with their queries and carry out

- appropriate actions such as scheduling tour.

- Users will be able to converse with the Chat bot through

- voice or text commands and it will understand what the user

- $\quad$ is saying through natural language understanding processing

- provided through the integration of Dialog flow API.

- Define meaningful user groups/personas.

- Determine user goals.

- Map user groups, goals and system actions.

\section{Non-Functional Requiremnts:}

The product should be open-source.

- The chatbot must be efficient with very little lag in response

- time for instance no longer than 5 seconds to reply to a user

- message.

- Talking to the bot should feel like talking to a person.

- The chatbot must be reliable with next to no faults or bugs.

- The database must be scalable to adopt to a growing

\section{STUDY OF EXISTING SYSTEM}

In existing system, chatbot takes input in the form of text command through chatbot application, analyses that input using Natural Language Processing techniques to find out what the user is trying to say or ask and responds accordingly. The travel chatbot is built to take all necessary inputs from the user to predict the relevant and accurate answers to the 
users. The chatbot will help the user for tourist purpose. The bot tells about the nearby places like restaurants, hotels.In the existing system the user needs to book tickets by himself. The user has to do through a login form, enter details, or book tours and travels serviceS This long and manual process becomes very difficult and time consuming. Lots of planning is required for traveling.

\section{PROPOSED SYSTEM ARCHITECTURE}

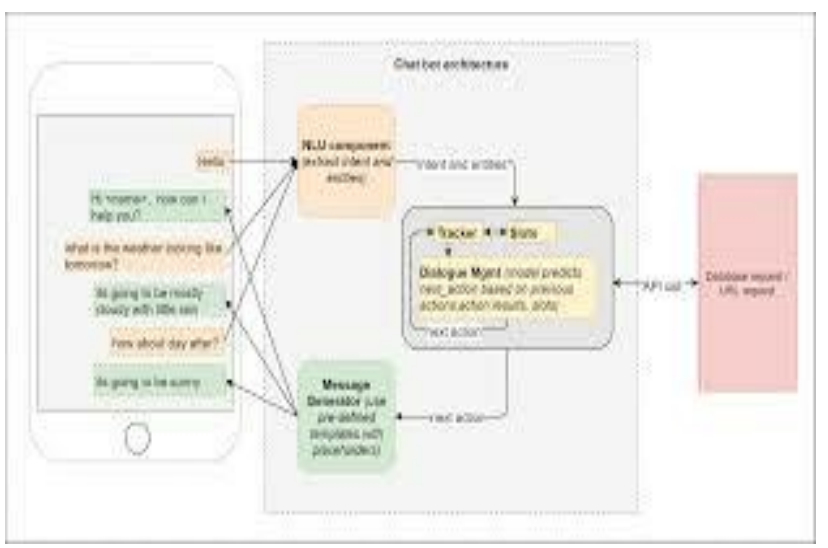

Fig 1 Proposed Architecture

\section{Detailed Architecture}

Its a chat-bot For simplicity reasons in this article, it is assumed thatthe user will type in text and the bot would respond back with an appropriate message in the form of text (So, we will not beconcerned with the aspects like ASR, speech recognition, speech totext, text to speech etc., Below architecture can anyways beenhanced with these components, as required).

It is conversational What does this mean? The bot should somehow maintain the state of the conversation and respond to the user request in the current

context.

- User - what is the weather looking like tomorrow?

- Bot - it's going to be mostly cloudy with little rain.

- User - how about the day after? (So, when this question is

posed, the bot should remember that the recent context of this conversation is regarding the entity "weather" (and not something else like a traffic congestion or a reservation booking availability, assuming they were present in the previous conversations).

It is an AI / ML driven architecture: The model learns the actions based on the training data provided (unlike a traditional state machine-based architecture that is based on coding all the possible if-else conditions for each possible state of the conversation.)

\section{SYSTEM DESIGN}

The whole method of developing the artificial intelligence based chatbot is explained in detail in this section. A developing procedure is divided into several important stages, including obtaining the user query, converting it into machine language, responding to the query in voice.

\section{Main Modules.}

Voice Recognition: For intelligent voice assistant application is done using Google Server. This process involves the conversion of acoustic

speech into a set of words and is performed by software component.

Accuracy of speech recognition systems differ in vocabulary size andconfusability, modality of speech (isolated, discontinuous, or

continuous speech, read or spontaneous speech), task and language constraints. The system consists of five modules: feature extraction, phone model training, dictionary preparation, grammar estimation, and

sentence decoding.

TTS Engine: A text-to-speech (TTS) system converts normal language text into speech. Synthesized speech can be created by concatenating

pieces of recorded speech that are stored in a database. The output is given in the form of speech.

Voice Input Manager: It manages the command given by user. It sends the Input given by user to the database manager, Database Manager: It compares Input given by user that is in the form of voice with the

database which contains vocabulary of words. It sends response to the action performer.

\section{DATASET}

\{"intents": [

$\{$ "tag": "greeting",

"patterns": ["Hi", "How are you", "Is anyone there?", "Hello", "Good day"],

"responses": ["Hello, thanks for visiting", "Good to see you again", "Hi there, how can I help?"],

"context_set": ""

\}

$\{$ "tag": "goodbye",

"patterns": ["Bye", "See you later", "Goodbye"],

"responses": ["See you later, thanks for visiting", "Have a nice day", "Bye! Come back again soon."]

\}

\{ "tag": "thanks",

"patterns": ["Thanks", "Thank you", "Thats helpful"],

"responses": ["Happy to help!", "Any time!", "My pleasure"]

\}

\{ "tag": "beach",

"patterns": ["maharashtra beaches", "pune 100005 days yes AC beach 3 people","pune 130006 days yes AC beach 4 people","pune 140006 days yes AC beach 4 people"],

"responses": ["Day 1, Alibaug <br> Alibaug is a dainty little beach town that is perfect for a three-day trip or a weekend getaway. On your first day here indulge in water 
sports or camel or bike rides at the Alibaug and Nagaon beaches. The next day go exploring the important Kolaba and Murud-Janjira forts and end the day with a nice local fish or vegetarian thali at Hotel Sanman, one of the most famous eateries in Alibaug. On day three, head about 32 kilometres away to Kashid Beach where you can enjoy surfing and spot a few dolphins. $\langle$ br $>\langle$ br $>$ Day 2, Dahanu-Bordi Beach $\langle$ br $>$ Located along the Thane coastline, the Dahanu-Bordi Beach is a calm and serene beach that is not yet explored to its fullest. Ideal for a three day trip from Mumbai, here you can go trekking, explore the pristine beaches and even enjoy a few water sports. Since the beach is located about a three-hour drive away from Mumbai, you have enough time to explore the beach town in two days; you can save the third day to just relax and unwind in nature's company before you get back to the grind. $\langle$ br $><$ br $>$ Day 3, Ganpatipule $<$ br $>$ The drive to Ganptipule takes about seven hours, so to reach by mid-day, you will have to leave Mumbai early in the morning. On your first day, you're bound to be a little tired, so do some light walking around villages to experience local culture. $\ln \backslash n \mathrm{nOn}$ the second day, head out to the beach and spend the whole morning swimming or just relaxing by the beach side. Save the third day for visiting one of the most important temples in Maharashtra, the Swayambhu Ganesh Temple. While you're there, enjoy local delicacies at one of the many restaurants near the temple. $\langle$ br $\rangle\langle$ br $\rangle "]$

$$
\} \text {, }
$$

\{"tag": "beach1",

"patterns": ["maharashtra beaches", "pune 100004 days yes luxury beach 2 people","pune 130006 days yes luxury beach 3 people","pune 140004 days yes luxury beach 3 people"],

"responses": ["Day 1, Alibaug <br> Alibaug is a dainty little beach town that is perfect for a three-day trip or a weekend getaway. On your first day here indulge in water sports or camel or bike rides at the Alibaug and Nagaon beaches. The next day go exploring the important Kolaba and Murud-Janjira forts and end the day with a nice local fish or vegetarian thali at Hotel Sanman, one of the most famous eateries in Alibaug. On day three, head about 32 kilometres away to Kashid Beach where you can enjoy surfing and spot a few dolphins. $\langle$ br $\rangle\langle$ br $\rangle$ Day 2, Dahanu-Bordi Beach $\langle$ br $\rangle$ Located along the Thane coastline, the Dahanu-Bordi Beach is a calm and serene beach that is not yet explored to its fullest. Ideal for a three day trip from Mumbai, here you can go trekking, explore the pristine beaches and even enjoy a few water sports. Since the beach is located about a three-hour drive away from Mumbai, you have enough time to explore the beach town in two days; you can save the third day to just relax and unwind in nature's company before you get back to the grind. $<$ br $><$ br $>$ Day 3, Ganpatipule $<$ br $>$ The drive to Ganptipule takes about seven hours, so to reach by mid-day, you will have to leave Mumbai early in the morning. On your first day, you're bound to be a little tired, so do some light walking around villages to experience local culture. $\ln \backslash n O n$ the second day, head out to the beach and spend the whole morning swimming or just relaxing by the beach side. Save the third day for visiting one of the most important temples in Maharashtra, the Swayambhu Ganesh Temple. While you're there, enjoy local delicacies at one of the many restaurants near the temple. $\langle$ br $>\langle$ br $>$ "]

\} $\{$ "tag": "beach2",

"patterns": ["maharashtra beaches", "pune 100004 days yes Deluxe beach 2 people","pune 130006 days yes Deluxe beach 3 people","pune 140004 days yes Deluxe beach 3 people"],

"responses": ["Day 1, Alibaug <br> Alibaug is a dainty little beach town that is perfect for a three-day trip or a weekend getaway. On your first day here indulge in water sports or camel or bike rides at the Alibaug and Nagaon beaches. The next day go exploring the important Kolaba and Murud-Janjira forts and end the day with a nice local fish or vegetarian thali at Hotel Sanman, one of the most famous eateries in Alibaug. On day three, head about 32 kilometres away to Kashid Beach where you can enjoy surfing and spot a few dolphins. $\langle$ br $>\langle$ br $>$ Day 2, Dahanu-Bordi Beach $\langle$ br $>$ Located along the Thane coastline, the Dahanu-Bordi Beach is a calm and serene beach that is not yet explored to its fullest. Ideal for a three day trip from Mumbai, here you can go trekking, explore the pristine beaches and even enjoy a few water sports.Since the beach is located about a three-hour drive away from Mumbai, you have enough time to explore the beach town in two days; you can save the third day to just relax and unwind in nature's company before you get back to the grind. $<$ br $><$ br $>$ Day 3, Ganpatipule $<$ br $>$ The drive to Ganptipule takes about seven hours, so to reach by mid-day, you will have to leave Mumbai early in the morning. On your first day, you're bound to be a little tired, so do some light walking around villages to experience local culture. $\ln \backslash n$ On the second day, head out to the beach and spend the whole morning swimming or just relaxing by the beach side. Save the third day for visiting one of the most important temples in Maharashtra, the Swayambhu Ganesh Temple. While you're there, enjoy local delicacies at one of the many restaurants near the temple. $\langle$ br $\rangle\langle$ br $\rangle "]$

\}, $\{$ "tag": "fort",

"patterns": ["fort in pune","pune 100005 days yes AC fort 3 people","pune 130006 days yes AC fort 4 people","pune 140006 days yes AC fort 4 people"],

"responses": ["1.raigad fort, 2.Pratapgad fort, 3.Sinhgad fort, 4.Murud Janjira fort,5.Panhala fort,6.Shivneri fort,7.Sindhudurg fort,8.Torana fort, 9.Daulatabad fort, 10.Lohgad fort "]

\section{\}}

\{"tag": "fort1",

"patterns": ["fort in pune","pune 100004 days yes luxury fort 3 people","pune 130006 days yes luxury fort 3 people","pune 140004 days yes luxury fort 3 people"],

"responses": ["1.raigad fort, 2.Pratapgad fort, 3.Sinhgad fort, 4.Murud Janjira fort,5.Panhala fort,6.Shivneri fort,7.Sindhudurg fort,8.Torana fort, 9.Daulatabad fort, 10.Lohgad fort "]

$\{$ "tag": "fort2",

"patterns": ["fort in pune","pune 120005 days yes Deluxe fort 3 people","pune 130006 days yes Deluxe fort 3 people","pune 140005 days yes Deluxe fort 3 people"], 
"responses": ["1.raigad fort, 2.Pratapgad fort, 3.Sinhgad fort, 4.Murud Janjira fort,5.Panhala fort,6.Shivneri fort,7.Sindhudurg fort,8.Torana fort, 9.Daulatabad fort, 10.Lohgad fort "]

$$
\}
$$

\{"tag": "Hill Station",

"patterns": ["Hill Station in pune", "pune 100005 days yes AC hill station 3 people", "pune 110006 days yes AC hill station 3 people","pune 100004 days yes AC hill station 3 people"],

"responses": ["A gorgeous hill station near Pune, Lonavala is located at the height of $622 \mathrm{~m}$ in the Sahyadri mountain range that separates the Deccan plateau and the Konkan coast. One of the most visited and favoured weekend getaways for people in Mumbai and Pune, this hill retreat boasts of caves, forts, lakes, and waterfalls amidst dense timberlands and verdant greenery. It's also known for its variety of 'Chikki.'"]

\}

\{"tag": "Hill Station1",

"patterns": ["pune 120005 days yes luxury hill station 3 people", "pune 150006 days yes luxury hill station 3 people","pune 100004 days yes luxury hill station 3 people"],

"responses": ["A gorgeous hill station near Pune, Lonavala is located at the height of $622 \mathrm{~m}$ in the Sahyadri mountain range that separates the Deccan plateau and the Konkan coast. One of the most visited and favoured weekend getaways for people in Mumbai and Pune, this hill retreat boasts of caves, forts, lakes, and waterfalls amidst dense timberlands and verdant greenery. It's also known for its variety of 'Chikki.'"]

\} ,

\{"tag": "Hill Station2",

"patterns": ["pune 100005 days yes Deluxe hill station 3 people", "pune 110006 days yes Deluxe hill station 3 people","pune 100004 days yes Deluxe hill station 3 people"],

"responses": ["A gorgeous hill station near Pune, Lonavala is located at the height of $622 \mathrm{~m}$ in the Sahyadri mountain range that separates the Deccan plateau and the Konkan coast. One of the most visited and favoured weekend getaways for people in Mumbai and Pune, this hill retreat boasts of caves, forts, lakes, and waterfalls amidst dense timberlands and verdant greenery. It's also known for its variety of 'Chikki.'"]

$$
\text { \} }
$$

\}

\section{CONCLUSION}

The purpose of a chatbot system is to simulate a human conversation. Its architecture integrates a language model and computational algorithm to emulate information online communication between a human and a computer using natural language. The low interaction time reflects the high understanding and speech recognition rates, offered through the adoption of conversational user interfaces thus allowing users to freely interact with the chatbot to meet the demands of modern life. The chatbot has proven to fulfil the demand of users wanting instant access and availability information and services.

\section{REFERENCES}

[1] Prof. Rakhi Bhardwaj, Poonam Gupta, Pooja Jadhav,

Bhagyashree Kadam, Amruta Kedari, "Android Based Automated Smart Wheel Chair", International Journal of Innovative Research in Computer and Communication Engineering, vol 4, Issue 3, March 2016.

[2] Kiran N.V, Madhan Mohan Reddy, Deepika Yadav, Babitha K.M, "Optimal driving system for two wheelers", Proceedings of IRF International Conference, 05th April-2014

[3] Yu Zhong, T. V. Raman, Casey Burkhardt, Fadi Biadsy and Jerey P. Bigham, "JustSpeak: Enabling Universal Voice Control on Android", April 2014

[4] Norhazahbt Aripin, M. B. Othman, "Voice control of home appliances using Android", Electrical Power, Electronics, Communications, Controls, and Informatics Seminar (EECCIS), 2014

[5] ArunGopi, Shobana Devi P, Sajini T, Bhadran V K, "Implementation of Malayalam Text to Speech Using

Concatenative Based TTS for Android Platform", International Conference on Control Communication.

\section{VIII.APPLICATION}

- It Can be Used for planning trip.

- It Can be used for booking Train, Bus, Cabs, Hotels. 